\title{
Der KÜNSTLER
}

\author{
ALS HOMO POLITICUS - DIE NARRATIVEN \\ GEMÄLde VON Simon Rosenthal
}

\section{The artist as Homo politicus - The narRative PAINTINGS OF SimON ROSENTHAL}

\author{
Karin MÜller-Kelwing
}

Staatliche Kunstsammlungen Dresden

Recibido: 31/03/2017 Evaluado: 04/05/2017 Aprobado: 30/10/2017

RESUMEN: Los cuadros narrativos del artista alemán Simon Rosenthal conforman una posición excepcional y enérgica de la pintura contemporánea figurativa. Surgen del enfrentamiento intelectual con nuestro tiempo, se originan por los actuales discursos sociales y reflexionan sobre la actualidad. Las obras del artista que vive en Dresde desconciertan al espectador en cuanto a la forma y al contenido. Muy a menudo no se ajustan a las expectativas. Las ambivalencias y las ambigüedades son intencionadas. Conscientemente, sus obras niegan una localización espacial y temporal. Imaginan una cierta atemporalidad y son imágenes con un subtexto político.

Refiriéndose a la historia cultural, el artista crea su propio mundo pictórico en el que los temas europeos y el cuestionamiento del poder juegan un papel importante. Las obras presentadas muestran a Rosenthal como «homo politicus».

Palabras claves: Simon Rosenthal, artista, pintura figurativa, pinturas narrativas, subtexto político

AвSTRACT: The narrative paintings of the young German artist Simon Rosenthal, who lives in Dresden, mark an extraordinary and strong position in the field of contemporary figurative painting.

Arising from the intellectual examination of our time, they ignite at contemporary discourses, always reflecting the now. Containing 
multiple irritations in form and content, they deliberately break with the viewer's expectations in many ways, evoking all kinds of ambiguities and ambivalence.

Negating any regional or temporal classification, Rosenthal's works conjure up a sphere above time, and may be looked at as a profound sort of political riddles.

Referring to cultural history, he creates a unique figurative cosmos, where European themes are raised, closely linked with the question of power. The presented works show the artist Simon Rosenthal as «homo politicus».

Keywords: Simon Rosenthal, artist, narrative paintings, figurative painting, political subtext

Zusammenfassung: Eine außergewöhnliche wie kraftvolle Position der zeitgenössischen figurativen Malerei bilden die narrativen Gemälde von Simon Rosenthal. Sie entspringen der intellektuellen Auseinandersetzung mit unserer Zeit, entzünden sich an aktuellen gesellschaftlichen Diskursen, reflektieren das Jetzt. Die Werke des in Dresden lebenden Künstlers enthalten inhaltliche und formale Irritationen, brechen mehrfach mit Erwartungen. Ambivalenzen und Ambiguitäten sind gewollt. Eine räumliche und zeitliche Verortung verweigern seine Werke bewusst, sie imaginieren Überzeitlichkeit, sind politische Denkbilder.

Unter Bezugnahme auf die Kulturgeschichte schafft er eine eigene Bilderwelt, in der europäische Themen und die Frage nach Macht eine bedeutende Rolle spielen. Die vorgestellten Arbeiten zeigen Rosenthal als «homo politicus».

$\mathrm{D}$ u musst Dein Leben ändern - so lässt der deutsche Dichter Rainer Maria Rilke 1908 sein Gedicht «Archaischer Torso Apollos» ${ }^{1}$ enden. Beim Anblick des nur fragmentarisch erhaltenen antiken Kunstwerkes hatte er entdeckt, dass diesem ein lebendiger Impuls, eine überraschende Aktualität inne wohnt.

Kunst wie Gesellschaft sind durchzogen vom Wunsch nach Veränderung, nach gesellschaftlichem Wandel, im zweiten Jahrzehnt des 21. Jahrhunderts stärker denn je. Zugleich herrscht große Irritation: Wie kann man heute

1. Rainer Maria Rilke: «Archaïscher Torso Apollos», in Rainer Maria Rilke: Der neuen Gedichte anderer Teil, Insel-Verlag, Leipzig, 1918, S. 1. Siehe: austrian literature online, http://www.literature.at/ viewer.alo?objid=12096\&viewmode=fullscreen \&scale=3.33\&rotate $=\&$ page $=3$ (01.08.2017). 
als Künstler arbeiten, wie sich in gesellschaftliche Prozesse einbringen, wie Menschen mit seinen Werken erreichen? Letztlich stellt sich die Frage, ob Kunstwerke mehr sind als Ab-Bilder, mehr als Träger von Erinnerungen und Hoffnungen? Können sie in Machtgefüge eingreifen, zum ethischen Handeln auffordern?

Die junge Künstlergeneration befindet sich im Aufbruch. Sie sucht nach neuen Formen und Ausdrucksweisen, jenseits der radikalen Abstraktion wie auch jenseits des Fotorealismus und des ideologiebestimmten Realismus des 20. Jahrhunderts. Einige entscheiden sich für die Flucht in ein apolitisches Werk. Sie kapitulieren vor der Angst, in ideologisches Fahrwasser zu geraten und kaum Verständnis beim Publikum zu finden. Nur wenige sind entschlossen, sich mit dem aktuellen Zeitgeschehen auseinanderzusetzen, eigene Gedanken und Zweifel in Kunst zu transformieren.

Über diese Tendenz konnte auch die weltweit bekannteste zeitgenössische Kunstausstellung, die documenta 14, nicht hinwegtäuschen, die 2017 in Kassel und Athen zahlreiche Arbeiten präsentierte, die sich mit politischen Themen wie Klimawandel, Rassismus, Armut, Flucht auseinandersetzen. Die ausgestellten Überreste havarierter Flüchtlingsbote wirken wie ein memento mori, lassen jedoch eine tiefere ästhetische wie intellektuelle Auseinandersetzung vermissen. Der als Wahrzeichen der documenta 14 bejubelte «Parthenon der Bücher» der argentinischen Künstlerin Marta Minujin verliert als Replik ihres bereits 1983 nach dem Ende der argentinischen Militärdiktatur gezeigten «Partenón de Libros» an Strahlkraft.

Angesichts der tagesaktuellen, gesellschaftlichen Realität, nicht nur in Sachsen, jener ach so fernen Region, von der weltweit primär über Intoleranz und Fremdenfeindlichkeit berichtet wird, stellt sich die Frage, ob sich Künstler unmittelbar in die gesellschaftlichen Debatten einmischen sollten. Oder sind es vielmehr andere Mittel und Wege, die junge Künstler heute reizen, sich in den aktuellen politischen Diskurs einzubringen?

Besonders deutlich wird dies angesichts der absurd anmutenden Diskussion um das im Februar 2017 von Manaf Halbouni in Dresden errichtete «Monument». ${ }^{2}$ Konzeptkunst hinterfragt, bisweilen plakativ, gewohnte Sichtweisen. So nutzt Halbouni eine Fotografie aus Aleppo und kombiniert diese mit anderen, bereits emblematisch gewordenen Erinnerungsbildern $\mathrm{zu}$ einer temporären Installation an prominentem Ort in Dresden - und provoziert: Neben großer medialer Aufmerksamkeit erntete er haltlose Beschimpfungen.

Willkommen im postfaktischen Zeitalter: Ertrinkend in einer InformationsFlut kapitulieren wir bereitwillig angesichts des Widerkäuens von Fake-News.

2. Siehe «Tumult auf dem Neumarkt», in Sächsische Zeitung, online, 07.02.2017 - http://www. sz-online.de/nachrichten/tumult-auf-dem-neumarkt-3607272.html (04.08.2017) und DoreEN ReINHARD: «Tumultartige Trauerarbeit», in Die ZEIT, 04.02.2017 - http://www.zeit.de/gesellschaft/ zeitgeschehen/2017-02/dresden-gedenken-13-februar-tumulte-einweihung-skulptur-monument-manafhalbouni (04.08.2017), auch https://www.manaf-halbouni.com/work/monument/ (04.08.2017). 
Macht und Gewalt - diese Themen und die entsprechenden Bilder umgeben uns täglich. Durch ihre rasante Verbreitung entwickeln sie eine Omnipräsenz, der wir uns kaum noch entziehen können. Doch mehr und mehr junge Künstler stellen sich dieser Herausforderung. Sie nehmen mit ihren Werken direkt oder indirekt Bezug auf diese Situation, auch und gerade auf die Gefahr hin, missverstanden und vehement abgelehnt zu werden - Entsteht ein Kunstwerk nicht gerade in diesem Spannungsfeld von Privatsphäre und gesellschaftlicher Realität?

Ein wichtiger Vertreter dieser mutigen, zeitgenössischen Künstlergeneration in Deutschland ist Simon Rosenthal. 1984 in Sulzbach-Neuweiler geboren und in Lüdenscheid aufgewachsen, studierte er Malerei und Grafik an der Hochschule für Bildende Künste in Dresden, seit 2016 ist er Meisterschüler bei Prof. Christian Macketanz.

Rosenthal entzieht sich bewusst der medialen Bilderflut. Er stellt sich gegen eine Kunst, die sich bereitwillig auf ihren Unterhaltungswert reduzieren lässt. Seine Bildthemen entspringen der intellektuellen Auseinandersetzung mit unserer Zeit, entzünden sich an aktuellen gesellschaftlichen Diskursen, reflektieren das Jetzt.

Besonders spürbar ist dies in seinen narrativen figurativen Gemälden, die seit etwa 2011 entstehen. Diese Arbeiten bewegen sich zwischen figurativem Realismus und narrativer Abstraktion. Sie sind nicht als Serie konzipiert, lassen sich aber sehr wohl als Werkgruppe lesen. ${ }^{3} \mathrm{Zu}$ den ersten narrativen Gemälden im Euvre Rosenthals, die sich insbesondere europäischen Fragestellungen widmen, zählen «Fähre» (Abb. 1) und «Abendstimmung» (Abb. 2), die sich beide heute in einer außereuropäischen Privatsammlung befinden. Etwa zeitgleich schuf der Künstler das Figurenbild «Soldat» (Abb. 3), gefolgt von «Star/Ego/USA (Der Wille zur Macht)» (Abb. 4). Ihren vorläufigen Höhepunkt finden Rosenthals erzählerischen Werke in den beiden äußerst komplexen, großformatigen Gemälden «Plateau Europa» (Abb. 5) und «Zeitenwende» (Abb. 6) von 2015-2016.

Aber sein Cuvre umfasst weitaus mehr als diese Werke, wie zum Beispiel zahlreiche Figurenbilder und Porträts. Längst ist der Künstler Simon Rosenthal kein Unbekannter mehr. Seit 2005 sind seine Arbeiten regelmäßig in Ausstellungen zu sehen, so in Bamberg, Berlin, Dresden, Erfurt, Leipzig, Lüdenscheid und Münster, aber auch bereits in Jeddah, London und

3. Diese Werkgruppe wird hier erstmals gemeinsam präsentiert. Die Idee zu diesem Aufsatz reifte während zahlreicher Gespräche im Atelier von Simon Rosenthal zwischen Oktober 2015 und August 2017, für die ich dem Künstler herzlich danke. Mein Dank gilt ebenso der Redaktion und den anonymen Gutachtern von POTESTAS für die aufmerksame Lektüre des Textes, ihre kritischen Anmerkungen und die Ermöglichung der Publikation.

4. Beide Gemälde stellte Rosenthal erstmals im Sommer 2016 aus. Siehe HoCHSCHULE FÜR BILDENDE KünSte (Hg.): Diplom 2016, Katalog zur Ausstellung, 15.07.-04.09.2016, Dresden, 2016 und Uwe SALzbrenNer: «Kunst kommt um den Spuk aus Europa nicht herum. Absolventen der Hochschule für Bildende Künste zeigen ihre Diplomarbeiten», in Sächsische Zeitung, 18.07.2016, S. 19. 
Yokohama. In mehreren privaten Kunstsammlungen ist er mit seinen Werken vertreten, auch außerhalb Europas. Rosenthal arbeitet mit Galerien in Berlin, Lüdenscheid und Stuttgart zusammen. In Dresden betreibt er gemeinsam mit

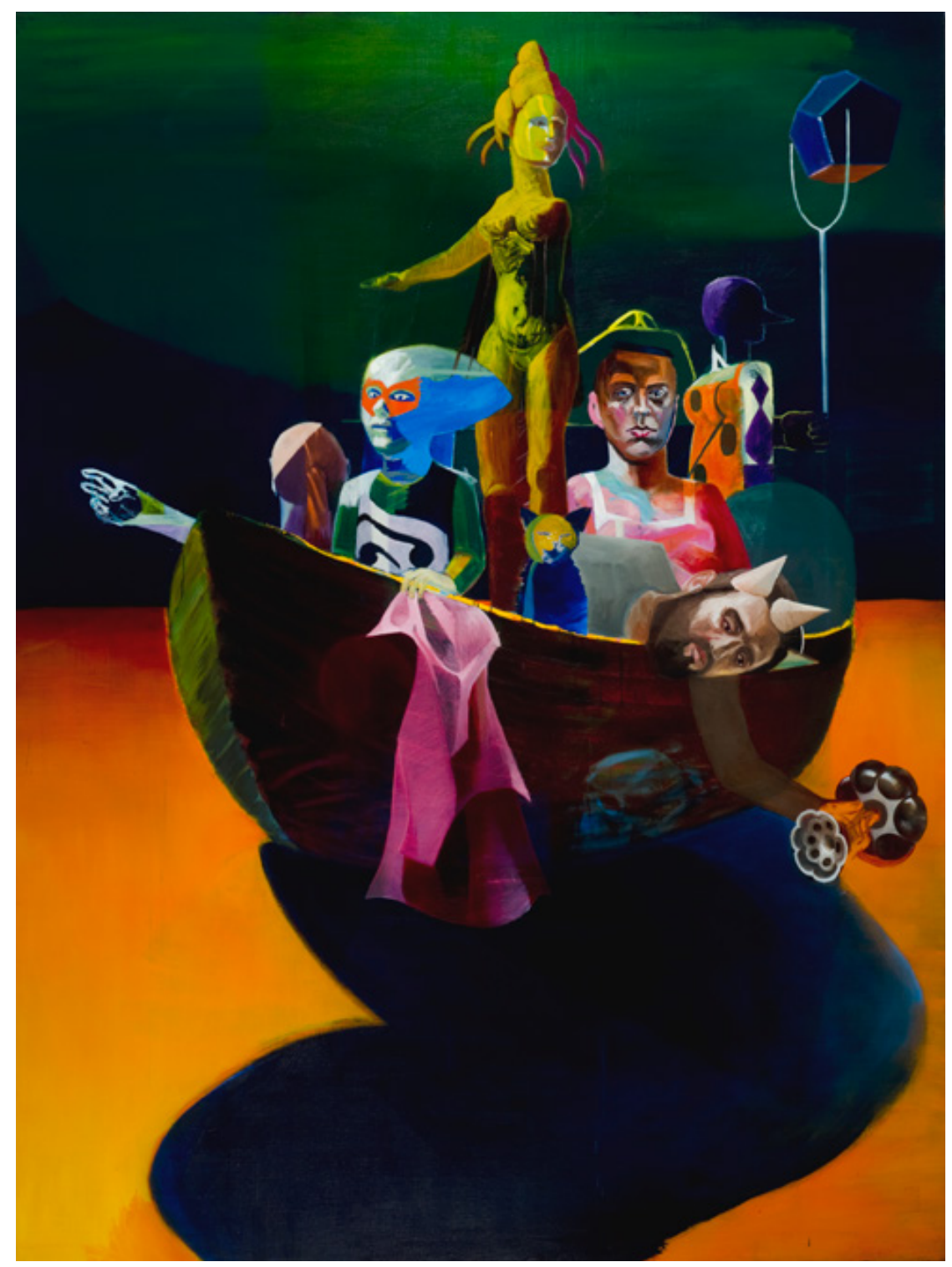

Abb. 1. Simon Rosenthal, Fähre, 2011-2013, Öl auf Leinwand, 240 x 180 cm,

$$
\text { Privatsammlung Saudi-Arabien }
$$




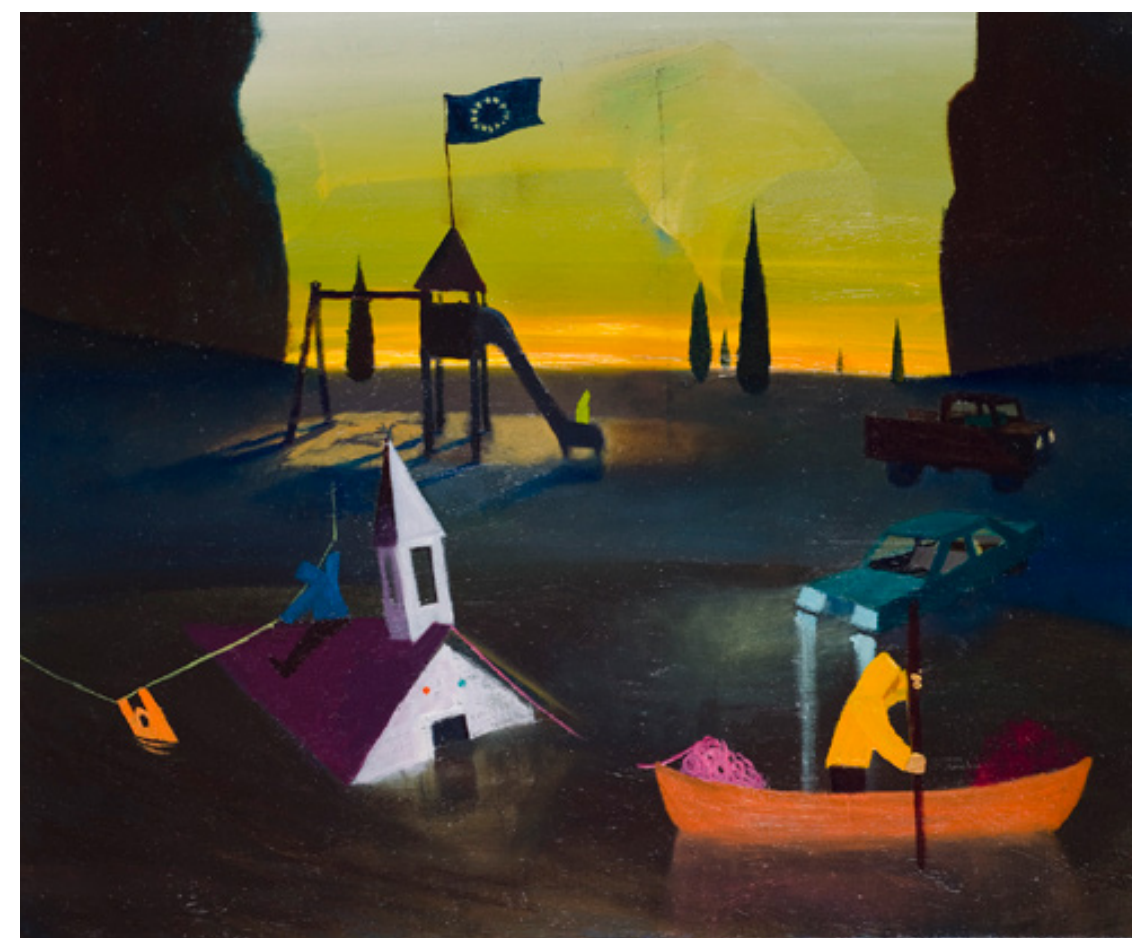

Abb. 2. Simon Rosenthal, Abendstimmung, 2012, Öl auf Leinwand, 50 x 60 cm, Privatsammlung Saudi-Arabien

neun anderen Absolventen der dortigen Kunstakademie die 2016 gegründete produzenten|galerie. ${ }^{5}$

Den Ausgangspunkt für seine prozessuale Arbeitsweise bildet das Sehen, die Anschauung. Das Naturstudium und vor allem die Arbeit mit lebendem Modell für seine Aktstudien und Porträts bedeuten dem Künstler viel. Doch sind sie für Rosenthal, wie Maisel bereits 2014 beobachtete, meist «nur ein Vehikel zum Einstieg in die aleatorische Bilderwelt» ${ }^{6}$.

Der lange Entstehungsprozess seiner Werke, der durchaus mehrere Monate, bisweilen, wie bei den großformatigen Gemälden, sogar Jahre dauern kann, beginnt relativ planlos. Erst beim Malen manifestiert sich unmittelbar auf der grundierten Leinwand eine erste vage Idee.

5. Siehe Karin Müller-Kelwing: «Ein neuer Ort für junge Kunst in Dresden. Zehn Künstler erfüllen sich ihren Traum», in Sachsenbummel. Magazin für KulturGeschichte und Tourismus, 23. Jg., Nr. 90, H. 1, 2016, S. 30-37. Siehe auch http://www.produzenten.net/ (10.08.2017).

6. Volker Maisel: «Vorwort», in Simon Rosenthal. Malerei und Grafik, Dresden, 2014, o. S. 


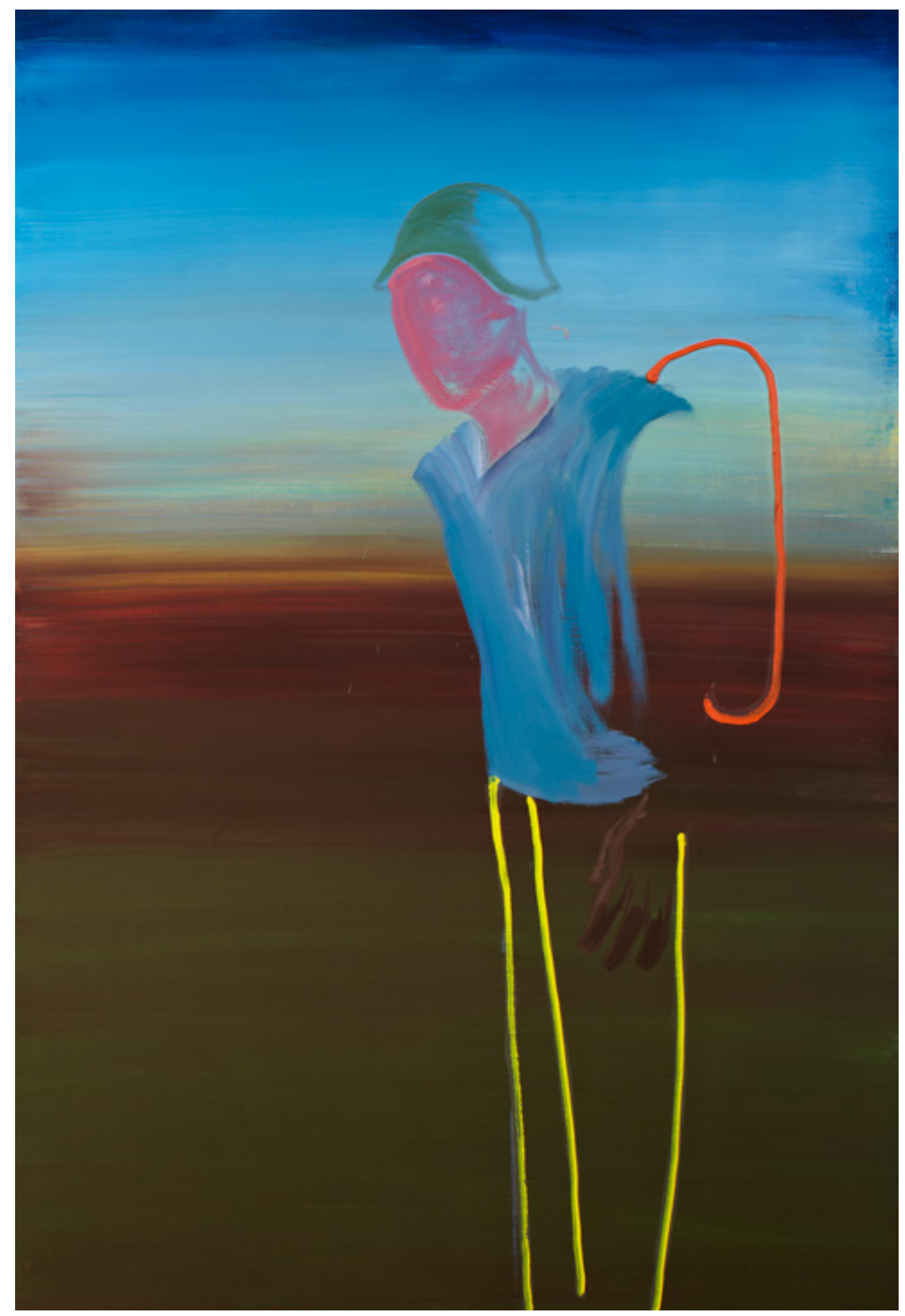

Abb. 3. Simon Rosenthal, Soldat, 2011, Öl auf Leinwand, 190 x 130 cm 


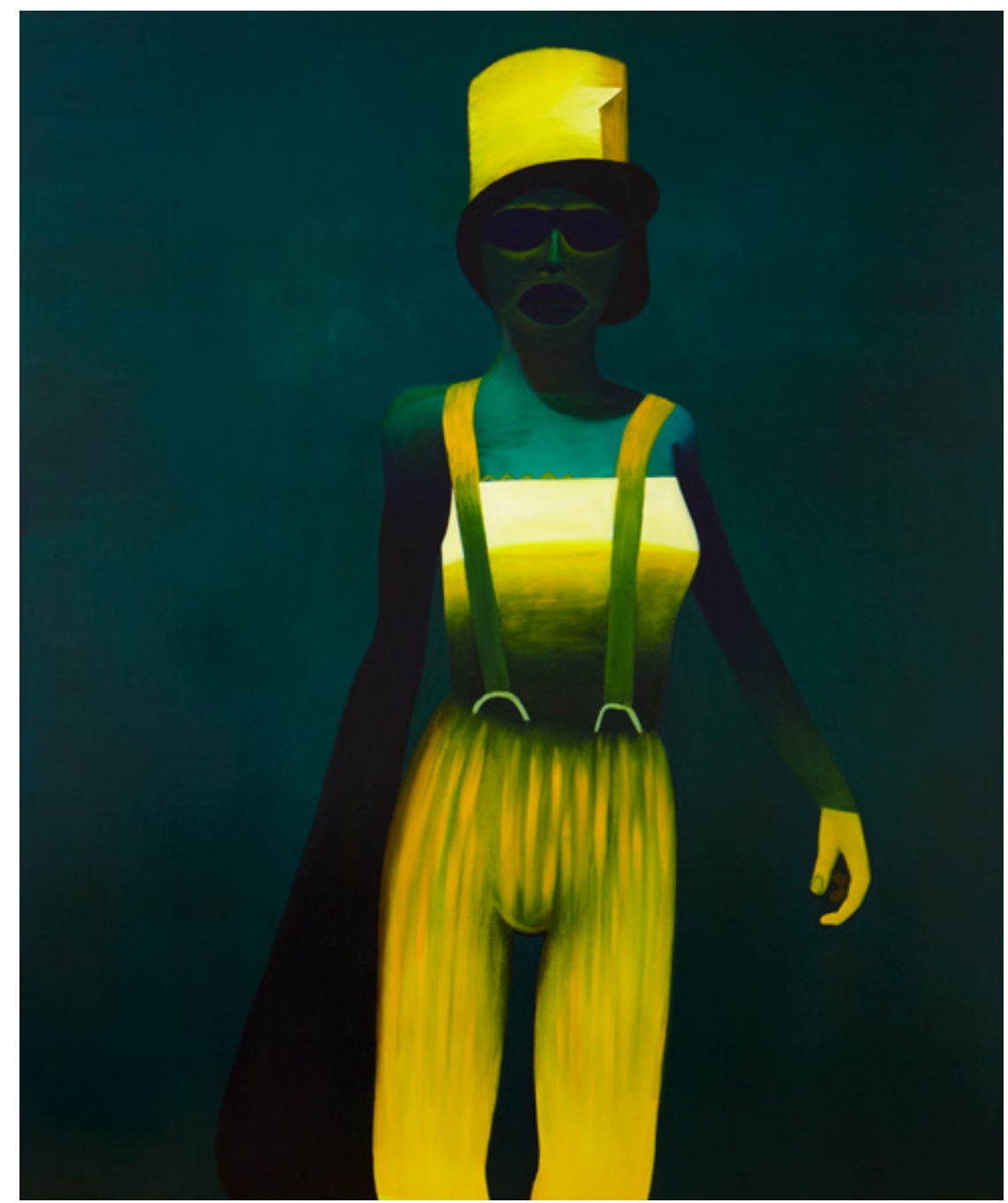

Abb. 4. Simon Rosenthal, Star/Ego/USA (Der Wille zur Macht), 2012-2014, Öl auf Leinwand, 180 x $150 \mathrm{~cm}$, Privatsammlung Deutschland

Der Akt des Malens dient dem Ergründen des eigenen Seins. Rosenthal spürt dabei den Schwingungen zwischen sich und seinem LeinwandGegenüber nach. Der Künstler führt gewissermaßen einen Dialog mit seinem entstehenden Werk, wobei die Leinwand in der künstlerischen Selbstbefragung den Part des Spiegels übernimmt. Sie wird zur Projektionsfläche für sein 


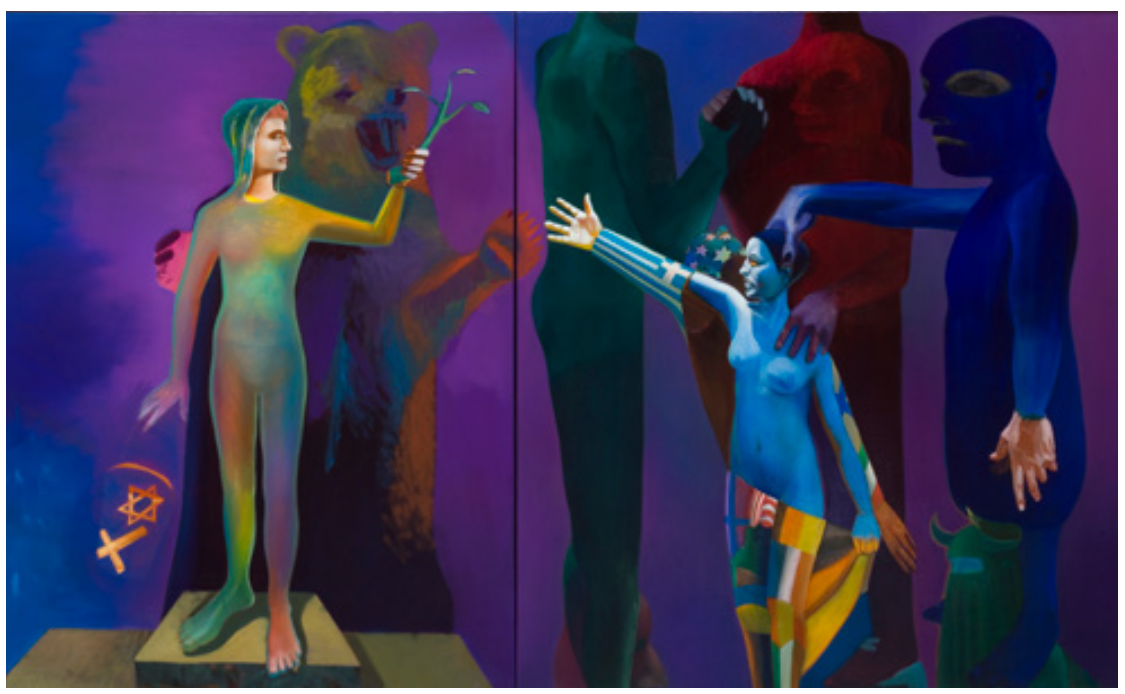

Abb. 5. Simon Rosenthal, Plateau Europa, 2015-2016, Öl auf Leinwand, 220 x 370 cm, Foto: Matthias Blumenhagen

Innerstes, für Gedanken und Gefühle, für Ängste, Wünsche und Hoffnungen. Jedes seiner Werke ist damit auch ein Stück weit Selbstporträt.

Dazu gesellt sich das Ringen um einen adäquaten formalen Ausdruck für diesen fragilen, sich ständig verändernden inneren Zustand. Erst nach und nach formt sich die Komposition, finden sich Formen und Farben auf der Leinwand. In diesem Arbeitsprozess lotet der Maler diverse Standpunkte aus, er verwirft Bildideen, findet neue. Das einzig Stete ist die Veränderung. Seine Gemälde «wachsen» - es handelt sich im wahrsten Sinne um eine BildFindung. ${ }^{7}$ Diese Suche führt über diverse Transformationen, die in formalen wie inhaltlichen Irritationen münden. Bisweilen verweigert Rosenthal die Zuordnung zu konkreten Formen, reduziert und verfremdet, löst die Figuration in verschiedenen Stufen der Abstraktion auf. Nicht selten steht in seinen Gemälden Hyperrealistisches unmittelbar neben Abstrahierendem. Insbesondere seine Personendarstellungen weisen formale Brüche auf, sind ihrer Individualität beraubt. Selbst in Porträts arbeitet er nie naturgetreu, auch sie weisen lediglich gewisse Züge von Realität auf. Jedoch ist der Abstraktionsgrad nicht von der Bedeutung des Dargestellten im Bildgefüge abhängig.

7. Zu seiner Arbeitsweise äußert sich der Künstler in Simon Rosenthal: In between realities, http:// artitious.com/artist/simon-rosenthal/ (06.08.2017). 


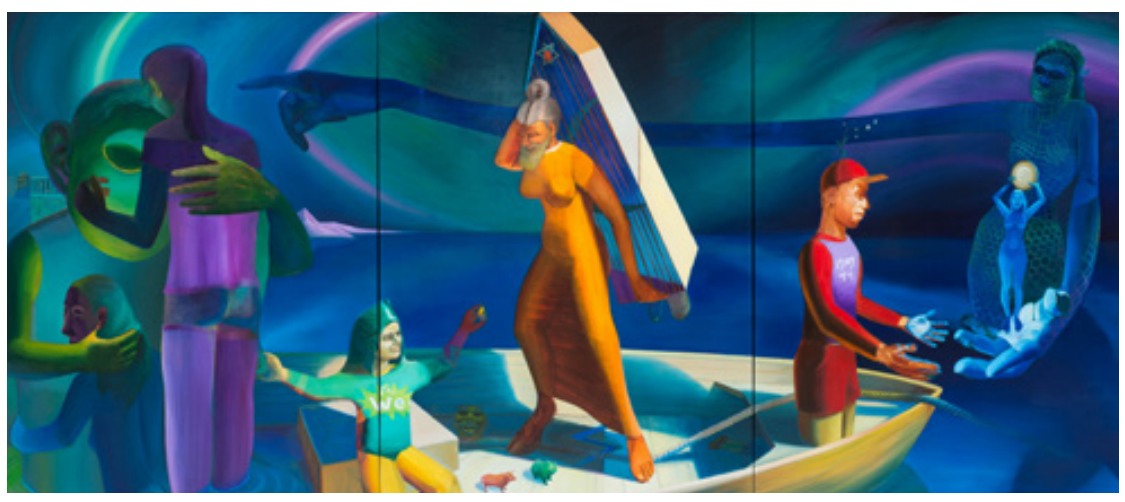

Abb. 6. Simon Rosenthal, Zeitenwende, 2015-2016, Öl auf Leinwand, 220 x $510 \mathrm{~cm}$, Foto: Robert Vanis

Die Frage, ob Malerei zeitgemäß ist, hat für Rosenthal keine Relevanz:

Form und Farbe, bewusst im Bild eingesetzt, sind als Ausdrucksmittel zeitlos. Sie sind ein visuelles Äquivalent unserer Gefühlswelt. In meinen narrativen Arbeiten sind die Inhalte zwar zeitbezogen, der bildnerische Schwerpunkt liegt jedoch in der Phänomenologie, ohne dass der Inhalt dabei weniger Bedeutung hätte. Dadurch verleihe ich meinen Bildern eine überzeitliche Dimension. Auf die Frage nach dem Zeitgemäßen habe ich immer in Paul Celans Gedicht eine Antwort gefunden: «Das Wort vom Zur-Tiefe-Gehn,/ das wir gelesen haben./ Die Jahre, die Worte seither./ Wir sind es noch immer./ Weißt du, der Raum ist unendlich,/ weißt du, du brauchst nicht zu fliegen,/ weißt du, was sich in dein Aug schrieb,/ vertieft uns die Tiefe. ${ }^{8}$

Simon Rosenthal hat in verschiedenen künstlerischen Techniken gearbeitet, bevorzugt jedoch die Malerei mit Ölfarben auf einer feinen, ebenmäßigen, hell grundierten Leinwand. Entgegen der aktuellen Moden entschied er sich bewusst für die altmeisterliche Technik der Lasurmalerei, wie sie in Dresden intensiv von Otto Dix und seinen Schülern, das heißt von den jungen Künstlern der 1920er Jahre, praktiziert wurde. ${ }^{9}$

8. Simon Rosenthal im Gespräch mit der Autorin am 20.03.2017. Das zitierte Gedicht entstammt dem Gedichtband «Niemandsrose» (1963). Siehe Beda Allemann und Stefan Reichert (Hg.): Paul Celan Gesammelte Werke in sieben Bänden, Suhrkamp, Frankfurt/Main, 1983, Bd. I, S. 211-291, hier S. 212.

9. Siehe: Holger Peter SAupe: «Dix-Schüler. Nähe und Distanz», in Kunstsammlung Gera (Hg.): Dix-Schüler. Nähe und Distanz. Gemälde Druckgraphiken Zeichnungen, Katalog der Ausstellung, Kunstsammlung Gera, 01.07.-20.08.1995, Gera 1995, S. 7-13 und G. SöDER: «Dix an der Dresdner Kunstakademie», in ibidem, S. 39-46, besonders S. 43. Für eine detaillierte Analyse der Maltechnik von Dix siehe: Marlies Giebe und Maria Körber: «... weil Dix hier wie ein Alter Meister und dabei doch ganz er selbst geblieben ist. Maltechnische Studien zum Triptychon „Der Krieg“ von Otto Dix», in StaAtliche Kunstsammlungen Dresden (Hg.): Otto Dix. Der Krieg - Das Dresdner Triptychon, Katalog der 
Geduldig trägt Rosenthal die Farben in dünnen Schichten übereinander auf. Die Zeit, die zum Trocknen der Schichten notwendig ist, ermöglicht dem Künstler das stete kritische Hinterfragen seiner gemalten Weltsicht und erleichtert die prozessuale Arbeit, die das Verwerfen und Finden neuer formaler Bildlösungen einschließt. Darüber hinaus erzeugt die subtil reduzierte, in feinsten Nuancen abgestufte Farbigkeit der Lasurmalerei einen inneren Glanz, ein Strahlen, suggeriert Raumtiefe, schafft eine Distanziertheit und entrückt ins Surreale.

In diesem aufwendigen Entstehungsprozess seiner figurativen Werke vermischen sich die intuitive und zugleich rationale Herangehensweise des Künstlers, vermengen sich der bisweilen rauschhafte Schöpfungsdrang und ein feiner Ordnungssinn, finden apollinisches und dionysisches Prinzip zu einer Einheit zusammen. Seine Werke erkennt Rosenthal erst als beendet an, wenn sich im Zwiegespräch mit ihnen der Titel in Worte fassen lässt. Erst dann trägt er den Firnis auf, dessen spätere optische Wirkung er im gesamten Malprozess bereits im Voraus mit bedenkt. Bisweilen sind die Titel seiner Gemälde sehr einfach, oft suggestiv, manchmal humorvoll, aber nie erklärend. Vielmehr unterwandern auch sie Erwartungen, sorgen für Irritation.

Seine Motive findet der Künstler in der innovativen wie spielerischen Auseinandersetzung mit dem aktuellen Tagesgeschehen, beim Grübeln über Politik und Gesellschaft. Seine Werke widmen sich sehr komplexen Themen, spiegeln das geistige Klima unserer Zeit. Rosenthal widmet sich existentiellen Fragen, analysiert den Zustand Europas, hinterfragt Identitäten, thematisiert Zugehörigkeiten.

Für Rosenthals Entwicklung als Maler ist die Begegnung mit Werken der Neuen Wilden, die sich in den 1980 Jahren nicht scheuten, ihre subjektiven Ansichten in zumeist großformatige Bilder mit expressiver Malweise und kräftiger Farbigkeit zu transformieren, wichtig. Er bekennt:

«Mich faszinieren künstlerische Positionen, die sich trauen, aus der Aktualität heraus, Ereignisse darzustellen, die Zeitgeschichte thematisieren, noch bevor sie als solche bezeichnet wird. Es gehört viel Mut dazu, sich selbst an den Schreibtisch der Geschichte zu stellen, die Signifikanz geschichtlicher Ereignisse zu definieren und in einem Gemälde zur Historie zu erklären Dafür bewundere ich Immendorf. Er legt so viel offen: Heimat, Liebe, Identität - Begriffe, die in der Kunst der globalisierten Welt nicht mehr vorkommen. Immendorf hat mit Café Deutschland gezeigt, dass die äußeren Geschehnisse die Innere Welt formen und anders herum, dass es eine Metaphysik in der Existenz gibt. Die Stärke dieser Bilder liegt darin, dass sie nicht die Banalität des Aktuellen scheuen, trotzdem mystisch sind und sich an einen Betrachter wenden, der aus historischer Distanz immer noch den Maler als Menschen in seiner Zeit spürt.» ${ }^{10}$

Ausstellung, Staatliche Kunstsammlungen Dresden, Galerie Neue Meister Albertinum, Sandstein Verlag, Dresden, 2014, S. 219-251.

10. Simon Rosenthal im Gespräch mit der Autorin am 26.11.2015. 
Bilden bei Immendorfs Bilderzyklus (1977-1982) die deutschen Befindlichkeiten im Kalten Krieg das Leitmotiv, so ist es bei Rosenthal Europa in der Identitätskrise, einer Phase des Umbruchs.

Rosenthals Figuren sind Zeugen und Protagonisten zugleich, doch selbst als Beobachtende niemals passiv. Mit dem steten Wechsel zwischen vagen formalen Andeutungen und klar formulierten Bildinhalten visualisiert er Widersprüche. Dabei bedient er sich traditioneller Symbole und kreiert eigene Bildzeichen, entwickelt eine individuelle Ikonographie. Es ist das Spiel von Realität und Fiktion, was den Künstler reizt. In seinen Arbeiten verschwimmen die Grenzen dazwischen. Nichts ist, als was es sich ausgibt. Seine Gemälde beinhalten gezielte Provokationen und (Ent-)täuschungen. Dennoch spricht aus seinen Werken das «Prinzip Hoffnung»" ${ }^{11}$. Rosenthal arbeitet mit inhaltlichen Gegenüberstellungen. Personen werden zu Dingen. Ebenso nehmen Dinge, auch Ideen, menschliche Züge an, kommen in seinen Bildern als Personifikationen daher. Der Künstler nutzt Metaphern, verwendet Sprachbilder, entwickelt daraus eine eigene Formen- und Bildsprache.

Doch egal ob seine Arbeiten surreal, (alb)traumartig oder humorvoll daherkommen - Eines tun sie immer: Sie geben Denkanstöße. Es sind jene werkimmanenten Brüche, besonders die formalen, die nicht der Bedeutung der Bildaussagen folgen, mit denen es Rosenthal geschickt versteht, den Blick des Betrachters zu lenken. So beginnen seine Gemälde von selbst zu erzählen, wenn der Betrachter sich darauf einlässt. Dieses Phänomen beschrieb der Maler und Schriftsteller Wolfgang Hildesheimer in anderem Zusammenhang wie folgt: «Kein Kunstwerk beantwortet eine Frage, die nicht gestellt wurde ... Wohl aber suggeriert es dem Betrachter eine Frage, und wenn er diese richtig zu stellen weiß, antwortet es unfehlbar mit einer ewigen Wahrheit.» ${ }^{12} \mathrm{Ganz}$ in diesem Sinne fordern die Werke von Simon Rosenthal den Betrachter zum Zwiegespräch, sie verlangen ein dialogisches Sehen.

Es ist an uns, an jedem Einzelnen, das richtige Wort zu treffen, die entscheidende Frage zu formulieren - dann offenbart sich uns der Zauber der Welt, dann lassen sich Bilder lesen. So gesehen, stehen Rosenthals Arbeiten in der romantischen Tradition, sie bedienen sich des Prinzips der Eichendorffschen Wünschelrute ${ }^{13}$. Doch Rosenthal verklärt nicht, der Künstler betrachtet die Gesellschaft mit analytischer Schärfe, transformiert seine kritischen Reflexionen in Bilder. Er fragt dabei nach politischer Involviertheit und hinterfragt gesellschaftliche Zusammenhänge. Er verwandelt die drängenden Fragen seiner Generation, auch jene nach der Zukunft Europas, in Malerei.

11. Vgl. Heinz Weissflog: «Das Prinzip Hoffnung. Malerei und Zeichnung von Simon Rosenthal in der produzentengalerie», in Dresdner Neueste Nachrichten, Nr. 286, 08.12.2016, S. 10.

12. Salman Ansari (Hg.): Wolfgang Hildesheimer - Die Schule des Sehens, Insel, Frankfurt/Main, 1997, o.S.

13. Siehe Joseph Freinerr von EichendorfF: «Wünschelrute», in Adalbert von Chamisso und Gustav Schwab (Hg.): Deutscher Musenalmanach für das Jahr 1838, Weidmannsche Buchhandlung, Leipzig, 1838, S. 287. Siehe https://www.uni-due.de/lyriktheorie/scans2/1838_eichendorff2.pdf (11.08.2017). 
Der Künstler ergründet in seinen Werken die Welt - will diese jedoch weder in ihrer Komplexität deuten, noch detailliert erklären. Vielmehr vermittelt er dem Betrachter eine vorsichtige Annäherung an die nun in seinem Bild enthaltene «Wahrheit», ohne dass diese komplett zu dechiffrieren wäre. Gerade in ihrer Doppeldeutigkeit entziehen sich Rosenthals Werke hartnäckig einer politischen Verwertbarkeit und ideologischen Instrumentalisierung.

Sein dialektisches Denken findet sein Pendant in der dialogischen Arbeitsweise. Die zahlreichen im Werk vorhandenen Bezüge und Anspielungen auf die Kunst- und Kulturgeschichte, wie auf die Epoche der Romantik oder die antiken Philosophen, überraschen wenig. Vor seiner Zeit an der Dresdner Kunstakademie studierte Rosenthal von 2004 bis 2009 an der Otto-Friedrich-Universität Bamberg Philosophie bei Roland Simon-Schaefer, sowie Kunstgeschichte, Restaurierungswissenschaften und Kunstdidaktik.

Rosenthal bedient sich poststrukturalistischer Instrumentarien. In seinen Gemälden vergegenwärtigt er die Foucaultsche Archäologie des Wissens und Ordnung der Dinge, ${ }^{14}$ lotet das Verhältnis zwischen künstlerischer Praxis und Wirklichkeit aus. Wie ein Palimpsest liegen philosophische Gedanken und literarische wie ikonographische Bezüge unter der sichtbaren Oberfläche seiner Gemälde verborgen.

In «Fähre» (2011-2013) schwingt unübersehbar der Bezug zur Romantik mit. (Abb. 1) Das Thema der Überfahrt, eines Überganges, entlehnt Rosenthal spielerisch bei Ludwig Richters «Überfahrt am Schreckenstein» $(1837)^{15}$. Scheinbar Überflüssiges, wie die Landschaft, lässt er weg, konzentriert sich ganz auf die Passage. Im Nirgendwo unterwegs, wird sein Boot zum Geisterschiff, gefüllt mit unterschiedlichen Charakteren. Der Gedanke an das «Narrenschiff» von Hieronymus Bosch" ${ }^{16}$ liegt nahe, ebenso jener zum gleichnamigen, 1494 gedruckten Buch von Sebastian Brant ${ }^{17}$. Sowohl Brant als auch Bosch hielten ihren Zeitgenossen einen Spiegel vor, schildern deren Laster und Eigenheiten. Ähnlich kritisch betrachtet Rosenthal die Gegenwart. Die gemeinsame Fahrt zielt ins Ungewisse. Daran wird auch der sich von den anderen Passagieren abwendende Nostalgiker, als Rückenfigur rechts im Boot dargestellt, nichts ändern. Formal angelegt wie die Identitätsfigur bei den Romantikern, ist es seine Aufgabe, uns als Betrachter ideell in das Bild zu integrieren: Bei allen gesellschaftlichen Veränderungen sitzen wir letztlich im selben Boot.

14. Siehe Michel Foucault: Archäologie des Wissens, Suhrkamp, Frankfurt am Main, 1973 (L'archéologie du savoir, 1969) und Michel Foucault: Die Ordnung der Dinge: Eine Archäologie der Humanwissenschaften, Suhrkamp, Frankfurt am Main, 1971 (Les mots et les choses: Une archéologie des sciences humaines, 1966).

15. Ludwig Richter, Die Überfahrt am Schreckenstein, 1837, Öl auf Leinwand, 116,5 x 156,5 cm, Staatliche Kunstsammlungen Dresden, Galerie Neue Meister, Gal.-Nr. 2229. Siehe https://skd-online-collection.skd. museum/Details/Index/311568 (21.08.2017).

16. Hieronymus Bosch, Das Narrenschiff, ca. 1494-1510, Öl auf Holz, 58 x 33 cm, Musée du Louvre, Paris, RF 2218. Siehe https://commons.wikimedia.org/wiki/File:Das_Narrenschiff.jpg (04.08.2017).

17. Siehe Sebastian Brant: Das Narrenschiff, Basel, 1494, Sächsische Landesbibliothek - Staats- und Universitätsbibliothek Dresden, Ink.394.4. Siehe http://digital.slub-dresden.de/werkansicht/cache.off?tx_ dlf\%5Bid\%5D=11823\&tx_dlf\%5Bpage\%5D=1\&tx_dlf\%5Bpointer\%5D=0 (04.08.2017). 
Die «Abendstimmung» (2012) ist bei weitem nicht so idyllisch, wie der Betrachter gemeinhin bei diesem Bildtitel erwarten dürfte. (Abb. 2) Im Streiflicht der untergehenden Sonne liegt zwischen zwei, bedrohlichen Schatten ähnlichen Felsen ein Spielplatz, auf dem die Europaflagge im Wind flattert. Friedlicher Platz, Insel, oder Kontinent? Nur noch eine Person scheint hier zu Rutschen. Ringsum, im Düsteren, bahnt sich eine Katastrophe an: Während das Wasser steigt, versuchen sich einige Personen zu retten. Zwei sind auf das Dach eines Gebäudes geklettert, das bereits in den Fluten versinkt - es könnte ein Rathaus sein, oder eine Dorfkirche. Eine weitere Person stakt in einem Kahn davon. Menschenleer, Geisterschiffen ähnlich, versuchen Autos, dem Unglück zu entkommen bzw. fahren direkt hinein. Inmitten der Sintflut ist kein Schutz zu finden. Europa vor dem Untergang? Alles scheint ins Wanken geraten, weder die Gesellschaft noch die Religion bieten Halt. Weltuntergangsstimmung. Nur die Natur scheint beständig. Trotzend ragen die an ihrer charakteristischen Wuchsform erkennbaren Lebensbäume, Symbole der Ewigkeit, in den orange-glühenden Himmel.

In «Star/Ego/USA (der Wille zur Macht)», 2012-2014, porträtiert Simon Rosenthal einen somnambulen Grenzgänger. (Abb. 4) Er ist Superheld und Chimäre in einem, flieht vor sich selbst. Er verführt und schockiert zugleich, gefangen im ewigen Kreislauf: Individuum und Gesellschaft, Macht und Ohnmacht, Schein und Sein, Leben und Tod.

Auf der Bühne des Lebens ein Star sein, Perfektion oder Illusion - ein kurzer Augenblick, der Moment des Innehaltens, des Nachdenkens. Einer Choreographie folgen, der eigenen oder der eines anderen, einen Schritt vor ins Rampenlicht, oder zwei zurück in den Schatten, hinter den Schatten unserer selbst. Das Hochgefühl des Erfolges suchen und ausleben, oder sich desillusioniert, gefangen, in uns selbst, verbergen. Den Höhenflug beginnen und genießen, oder ikarusgleich sich die Flügel verbrennen, abstürzen. Es geht ums Scheitern oder Gewinnen, um Selbst- oder Fremdbestimmung, um Macht und das Überwinden von Ängsten. Wie in anderen Gemälden Rosenthals auch, ist dem «Star» keine eindeutige geschlechtliche Rolle zugeordnet. Ist dies ein bewusstes Spiel mit Rollen, die uns in der Gesellschaft zugewiesen werden? Oder ist es gar ein Ausdruck der inneren Zerrissenheit der Gedanken und Gefühle? Der «Star» spielt mit Gegensätzen und spiegelt unser Ego. Doch was in diesem Gemälde noch als furchterregende, Sprachlosigkeit hervorrufende Vision erscheint und im Titel nur angedeutet ist, wurde inzwischen mit der Wahl von Donald Trump zum US-Präsidenten von der Wirklichkeit konterkariert.

Mit dem Titelzusatz «Der Wille zur Macht» verweist Rosenthal auf den vom Philosophen Friedrich Nietzsche geprägten Begriff, der ausdrücklich nicht auf die Herrschaft über andere zielt. ${ }^{18}$ Vielmehr verbirgt sich darin eine

18. Siehe Friedrich Nietzsche: Der Wille zur Macht. Versuch einer Umwertung aller Werte, Alfred Kröner Verlag, Leipzig, 1928. 
lebensbejahende Haltung, die beinhaltet, als Mensch sein Schicksal aktiv anzunehmen und in die eigenen Fähigkeiten zu vertrauen.

Rosenthals «Soldat» (2011) erscheint als gesichtsloser, gewaltbereiter Dienstleister im Nirgendwo. (Abb. 3) Viel ist nicht von ihm geblieben. Er verliert sich förmlich in der Umgebung. Seine Körperlichkeit ist nur angedeutet: Helm, Tornister, die Hand dienstbeflissen wie gehorsam an der Uniformnaht. Als Schatten seiner selbst steht er da - ohne Gesicht, identitätslos, im Dienst einer (Wirtschafts-)Macht zum Kämpfen verpflichtet. Ist er zerbrechlich oder längst gebrochen? Über das äußere Erscheinungsbild seines Postheroen kommuniziert der Künstler dessen «Zerbrechlichkeit, das Gebrochen- und Gehemmt-Sein des Kriegers unserer Tage - letztlich des Menschen im postheroischen Zeitalter». ${ }^{19}$ Ruhm hat keine Bedeutung mehr, Macht ist Selbsttäuschung und selbst die Liebe scheint unglaubwürdig geworden zu sein. ${ }^{20}$ Zugleich stellt sich die Frage nach der Individualität des Individuums in der Masse.

Ein weiteres Gemälde dieser Werkgruppe von Simon Rosenthal erzählt von der Suche nach Identität: In «Plateau Europa» (2015-2016) entsteigt eine junge Frau einem Kokon, oder hält diesen schutzbedürftig fest. (Abb. 5) Hier versucht Europa der Phrasenhülle der verkrusteten Nationalismen zu entkommen, die von der Gesellschaft vordefinierte Rolle abzulegen, oder verharrt, nackt und schutzlos, in der Depression gefangen, wie paralysiert. Die drei großen Figuren im Hintergrund konterkarieren ihre Bemühungen. Handelt Europa noch selbstbestimmt, oder wird es zurückgehalten, gar instrumentalisiert? Ist Europa fähig, sich weiter zu entwickeln, neue Wege zu gehen? Gelingt es, die Nationalismen abzulegen? Welche Werte sind uns wichtig? Durchtrennen wir unsere kulturellen Wurzeln allzu willfährig, wenn wir die Religion ablegen, wie jene schillernde Figur, die die Symbole dreier Religionen fallen lässt? Diese Geste und das gemeinsame Darstellen der Symbole von Judentum, Christentum und Islam verweisen in der Umkehrung an die Ringparabel bei Gotthold Ephraim Lessing ${ }^{21}$ und damit auf die Europäische Aufklärung. Nie zuvor waren wir so unfrei wie heute, obwohl unsere Freiheit scheinbar grenzenlos ist. Eine Rückbesinnung auf die Errungenschaften der Aufklärung tut not - Rosenthal steht damit nicht allein. Auch der Erzähler und Lyriker Jörg Bernig ${ }^{22}$ bezog sich in seiner 2016 in Kamenz gehaltenen Rede explizit auf deren noch immer aktuelles Gedankengut, auf die Freiheit des Denkens und der Meinung, als er Immanuel Kant zitierte: «Sapere aude! Habe Muth dich deines eigenen Verstandes zu bedienen!».23

19. SimON Rosenthal: Soldat, 2016, http://atelier-simon-rosenthal.de/de/texts/ (05.08.2017)

20. Siehe ibidem.

21. Gotthold Ephraim Lessing: Nathan der Weise. Ein dramatisches Gedicht, in fünf Aufzügen, Erstausgabe: Berlin 1779. Siehe http://gutenberg.spiegel.de/buch/nathan-der-weise-1179/3 (06.08.2017).

22. Siehe Jörg Bernig: Habe Mut ... Eine Einmischung, 3. Kamenzer Rede in St. Annen, im Rahmen der Lessing-Rezeption, Kamenz, 07.09.2016, http://www.nachdenkseiten.de/upload/pdf/161219-04Endfassung-J-Bernig-3-Kamenzer-Rede.pdf (06.08.2017).

23. ImMANuel Kant: «Beantwortung der Frage: Was ist Aufklärung?», in Berlinische Monatsschrift, 1784, H. 12, S. 481- 494, hier: S. 481, siehe http://www.deutschestextarchiv.de/book/view/kant aufklaerung_1784?p=17 (06.08.2017). 
Simon Rosenthal greift in «Plateau Europa» Mythen und Symbole auf, verwandelt sie in eigene Bildzeichen. Doch er folgt in diesem Gemälde nicht dem tradierten Europa-Mythos sondern reflektiert das Jetzt. Macht oder Ohnmacht? Bestandsanalyse oder Zukunftsvision? ${ }^{24}$ Dogmatik liegt dem Künstler dabei fern. Sein Fragen erweckt den Eindruck der kindlich-naiven Absichts- und Arglosigkeit, sein prüfender Blick bleibt unvoreingenommen und offen.

Sehr ähnlichen, aber im Grunde keinen rein europäischen Fragen geht Rosenthal im Gemälde «Zeitenwende» (2015-2016) nach. (Abb. 6) Auf rätselhafte Art mit einer höheren Macht verbunden sind jene sechs Personen, die, in einem Boot befindlich, auf der Flucht zu sein scheinen. Die Bilderzählung dominiert jedoch, aus dem Hintergrund agierend, eine moderne Sirene, ein blaues Wesen, von einem Polygonnetz überzogen, das in Fischschuppen endet. Dieses, der antiken Mythologie entlehnte, weibliche Fabelwesen, von dem Homer in seiner Odyssee berichtet, verkörpert bei Rosenthal die Personifizierung einer hochtechnisierten, virtuellen Welt. Lockten die Sirenen einst Odysseus mit ihrem Gesang und ihrer Fähigkeit, allwissend zu sein, so verfügt die Cyberwelt heute über eine ähnliche Anziehungskraft. Sie ist verheißungsvoll, verführt mit Versprechungen. Wie die Fischer einst zum Opfer ihrer Neugier wurden, so driften junge Menschen heute zusehends in die Cyberwelt ab, verlieren jedweden Bezug zur Realität, leben in der Virtual Reality, im SecondLife. Ist es dieser Realitätsverlust, der totalitäre Systeme erstarken lässt, weil viele Menschen haltlos geworden sind und den Wunsch verspüren, sich von einer Person leiten zu lassen, die ihnen Entscheidungen abnimmt, die uns das reale Leben tagtäglich abverlangt? Auch diese moderne Sirene - Maschine mit künstlicher Intelligenz, Meerjungfrau oder Göttin will verführen. Sie offeriert auf ihrer ausgestreckten geöffneten linken Hand eine wohlgeformte junge Frau mit einer überdimensional großen EuroMünze, letztlich ein Symbol für die erotische Ausstrahlung von Macht, Geld und Technik.

Doch mit einer anmaßenden Geste weist sie zugleich, gewissermaßen hintergründig, mit ihrer rechten Hand auf die kulturellen Wurzeln Europas, versinnbildlicht als Parthenon der Akropolis in Athen. Mahnt diese Geste zur Rückbesinnung, gar zum Umkehren, oder zeigt sie nur einen von vielen möglichen Wegen? In Verbindung mit dem Tau erinnern diese Gesten nicht nur an die Ballade «Der Fischer» von Johann Wolfgang Goethe ${ }^{25}$, sondern vielmehr an die Vielschichtigkeit der wissenschaftlichen und philosophischen Exkurse im Roman «Moby Dick» von Herman Melville ${ }^{26}$. Das Tau evoziert zugleich das Bild des Tauziehens, eines Kräftemessens. Gleichermaßen schafft

24. Siehe Karin Müller-Kelwing: «Simon Rosenthal: Plateau Europa», in Hochschule für BiLdende KüNSTE, Diplom 2016, S. 145.

25. Siehe https://de.wikisource.org/wiki/Der_Fischer (05.08.2017).

26. Herman Melville: Moby-Dick or The Wale, Harper \& Brothers Publishers, New York, 1851 (Erstausgabe), siehe http://www.gasl.org/refbib/Melville_Moby_Dick.pdf (05.08.2017). 
es eine Verbindung zwischen dem Boot mit seiner disparaten Besatzung und den Verlockungen unserer Zeit, fungiert als eine Art Nabelschnur - verbindend und alimentierend.

Der Gedanke an Identitätsverlust und Überwachung schwingt in dieser Darstellung mit, wie auch jener an George Orwells Überwachungsstaat: «Big brother is watching you» ${ }^{27}$. Auch in «Zeitenwende» untersucht der Künstler letztlich Machtstrukturen, lotet er die Mechanismen physischer wie psychischer Macht aus. Eine solche Macht bilden die allgegenwärtigen Neuen Medien, von denen wir uns willfährig dirigieren und manipulieren lassen. Ähnlich wie in «Star» und «Plateau Europa» thematisiert Rosenthal in «Zeitenwende» Versprechen und Betrug, Allmacht und Ohnmacht sowie Verführung und Manipulation. Dabei verzichtet er darauf, Gewalt darzustellen, ganz im Sinne des dem Psychiater und Konfliktforscher Friedrich Hacker zugeschriebenen Zitates: «Das Fehlen sichtbarer Gewalt erlaubt der Manipulation, sich als jene Freiheit auszugeben, die sie entzieht. $»^{28}$

Bei der Darstellung der Besatzung des Bootes pointiert Rosenthal wesentliche Aussagen seines Gemäldes, ästhetische Gesetzmäßigkeiten sind dabei zweitrangig. Das Kind im Boot ist jenes ewige Kind, das sich sträubt, erwachsen zu werden und Verantwortung zu übernehmen. Es wähnt sich sicher und geborgen, nimmt aber doch an einer Irrfahrt, einer Fahrt mit ungewissem Ausgang teil. Dieses Kind ist die einzige Figur im Boot, die mittels ihrer Körperhaltung und Gestik einen Bezug zwischen Dargestelltem und Betrachter herstellt, die Mittler sein will. Es scheint ein Abbild der Generation des Künstlers zu sein, Stellvertreter jener jungen Europäer, für die Frieden und materielle Sicherheit allzu selbstverständlich scheinen.

Selbst kleinste, zufällig wirkende, banale Details, wie der Aufdruck auf dem Shirt des Kindes in «Zeitenwende», entpuppen sich als bewusst platzierte, doppelbödige Aussagen. Da ist zu lesen The We - Wir, die Allgemeinheit - oder The West - der Westen, die westliche Welt, das Abendland. Aus zentraleuropäischer Perspektive heraus, ist dies als klarer Bezug zu Europa als Kern der westlichen Welt zu lesen.

Geradezu winzig erscheinen Bär und Bulle, als seien sie vom Kind absichtslos beim Spielen vergessen worden. Die Größe der im Bild dargestellten Attribute lenkt von deren Bedeutung ab. Im Sinne der Bedeutungsperspektive schwingt die Frage mit nach der tatsächlichen Bedeutung von Börsengeschäften, die durch diese Tiere symbolisiert werden, für und ihrem Einfluss auf den Fortgang der Welt.

Die einzige Person im Boot, die tatsächlich mit der Personifizierung der Macht interagiert, ist jene mit hyperrealistischen Gesichtszügen im roten Sportdress. Sie steht aufrecht, scheint fest mit dem Boot verwachsen, wie eine Galionsfigur. Auf ihrem Shirt können Japanisch-Kundige kawaii lesen:

27. George Orwell: Nineteen Eighty-Four, Penguin, London 2008 (Erstausgabe 1948).

28. Siehe https://de.wikipedia.org/wiki/Manipulation (05.08.2017). 
«niedlich «, «liebenswert». So wäre diese Person gern - anerkannt und von allen akzeptiert. Anderen zum Gefallen schmückt sie sich mit Blümchen. Es allen recht machen wollend, hat sie sich die Hände «schmutzig gemacht», wie deren Blaufärbung offenbart. Rührt dies vom bereitwilligen Annehmen der verlockenden Gaben her? Die Geste der nach oben geöffneten Hände erinnert zugleich an den Empfang eines Segens.

In der Mitte des Gemäldes und dennoch nicht als zentrale Figur steht ein androgynes Mischwesen: ein Bartträger mit weiblichen Brüsten, der sein langes Haar zu einem Knoten gebunden trägt. Unter dem langen orangen Gewand sind ein Huf und ein nackter Fuß sichtbar. Es ist ein Wesen ohne eindeutige Identität, jedoch in angedeuteter eiliger Bewegung, heimatlos sein Hab und Gut mit sich tragend, scheinbar nirgends zugehörig.

Interessant ist auch jene Gruppe von drei mit sich beschäftigten, sich umarmenden Personen links im Boot. Diese scheinen nur körperlich präsent zu sein, vom Geschehen um sich herum Nichts zu bemerken. Dazu im Gegensatz steht ihre, im Gemälde nur angelegte, abstrakte Körperlichkeit, die auf realistische Details verzichtet. Verbirgt sich hier ein Verweis auf das biblische Sodom und Gomorra, auf Lot und seine Töchter? Es ist kein Zufall, dass diese Gruppe im Wasser steht - offensichtlich hat das Boot ein Leck und droht unterzugehen.

Mit seinem Gemälde bekräftigt der Künstler, dass eine fundamentale Zeitenwende im Gang ist, aber er verweigert die Aussage, was diese Zeitenwende bedeutet, welche Auswirkungen sie für die Zukunft haben wird. Das Polarlicht im Hintergrund, als Urkraft der Natur, bietet eine mögliche Antwort. Es ruft das enorme Potential der Natur in Erinnerung und verweist darauf, wie klein und unbedeutend wir Menschen letztlich im Gesamtgefüge der Welt sind.

Die Beschäftigung mit dem Thema Europa in meinen Arbeiten entspringt der Beobachtung, dass die demokratischen Grundwerte Europas dem aktuellen politischen Geschehen mehr und mehr untergeordnet werden. Eine aktive Suche nach einer gemeinsamen europäischen Identität ist unerlässlich für unsere Zukunft. Überlassen wir diese Frage den Institutionen und Großkonzernen, dann scheitert Europa am Mangel einer gemeinsamen kulturellen Identität. Mit meinen narrativen Bildern bringe ich das Thema der europäischen Identität in die aktuelle Kunst ein. Sie stellen sowohl für Künstler als auch Rezipienten eine Herausforderung dar, die sich über die wahrnehmungstheoretischen und diskursiven Aspekte von bildender Kunst hinaus einen Blick auf das Ganze erarbeiten wollen. ${ }^{29}$

In den narrativen Werken von Simon Rosenthal begegnen sich, einer synoptischen Erzählung gleich, mehrere Welten. Realität und Fiktion, Frau und Mann, Macht und Ohnmacht, Individuum und Gesellschaft... Unzählige 
Dichotomien, sich bedingende, durchdringende, einander ergänzende und gegenseitig ausgleichende Prinzipien verbergen sich in diesen Gemälden. Dabei entstehende Ambivalenzen und Ambiguitäten sind gewollt. Sie bestimmen Simon Rosenthals Bilderwelt. Seine Werke enthalten inhaltliche und formale Irritationen, brechen mehrfach mit Erwartungen. Eine räumliche und zeitliche Verortung verweigern seine Werke bewusst. Sie imaginieren Überzeitlichkeit. Rosenthal erzählt, er

vermisse in der Kunst wie in der Gesellschaft, den Gedanken an die Ewigkeit, denn unsere Leitbilder sind nur noch auf kurze Zeit gedacht, bieten keinerlei Stabilität. Mit der formalen Ausprägung meiner Werke suche ich eine ästhetische Dimension, die länger hält, als das Thema zeitgemäß ist. ${ }^{30}$

Doch Rosenthal ist kein Theoretiker. Insbesondere die Gemälde «Plateau Europa» (Abb. 5) und «Zeitenwende» (Abb. 6) sind sein persönlicher Beitrag $\mathrm{zu}$ einer europäischen Verständigung. Seinen Bildern lässt der Künstler weitere Taten folgen und engagiert sich auch als Kulturvermittler für die Europäische Idee. So wird er diese Bilder Ende Januar 2018 im Europäischen Parlament in Brüssel präsentieren, gemeinsam mit Werken des in Berlin lebenden, gebürtigen Iraners Saeed Foroghi. Der Titel der Ausstellung, «Referendum», ist sogleich Programm. Bereits 2016 initiierte Rosenthal das Ausstellungsprojekt «Nähe und Distanz», bei dem Studenten der Fachklasse Macketanz der Hochschule für Bildende Künste Dresden mit Studierenden der Academy of Fine Arts Skopje zusammentrafen und gemeinsam in Skopje ausstellten. ${ }^{31}$

Längst hat Rosenthal für sich erkannt, dass es für eine Demokratie wichtig ist, dass Kunst sich an der Gesellschaft, aus der sie sich letztlich generiert, beteiligt. Er tut dies insbesondere mit seinen narrativen Gemälden, denen eine beharrliche wie «humoristische Ernsthaftigkeit» ${ }^{32} \mathrm{zu}$ eigen ist, und vertritt damit eine außergewöhnliche, eigenwillige wie kraftvolle Position der aktuellen figurativen Malerei. Seine Werke unterhalten und stiften Unruhe, indem sie unsere Sehgewohnheiten und Denkweisen konterkarieren, uns irritieren. Als Betrachter müssen wir das lediglich zulassen, dürfen wir uns diesem Zwiegespräch hingeben.

Bei der Eröffnung der Salzburger Festspiele 2016 stellte der Philosoph Konrad Paul Liessmann in seinem Plädoyer für die Kunst in unruhigen Zeiten fest:

30. Simon Rosenthal im Gespräch mit der Autorin am 01.08.2017.

31. Nähe und Distanz. Internationale Ausstellung Skopje - Dresden, 11.09.-17.09.2016, Skopje. Siehe https://www.youtube.com/watch?v=8-YfGPSxM-w (04.08.2017). Die anlässlich des 50-jährigen Jubiläums der Städtepartnerschaft Dresden - Skopje für 2017 geplante Fortsetzung des Projektes scheiterte leider an der Finanzierung.

32. MAISEL, Vorwort, o. S. 
Die Kunst erfordert, heute mehr denn je, das Eintauchen in eine andere Welt, eine Welt, in der es um Genauigkeit, Aufmerksamkeit, Konzentration, Hingabe, Anstrengung und Selbstvergessenheit geht, um Haltungen also, die quer stehen zu jener Mischung aus Bequemlichkeit und Egomanie, zu der wir ansonsten angehalten sind. ${ }^{33}$

Insbesondere mit seinen narrativen Gemälden stellt sich Simon Rosenthal dieser Herausforderung. In ihnen wagt er die Annäherung an die Widersprüche und Abgründe des Menschen. Seinen Werken wohnt eine zeitlose Aktualität inne. Diese erschließt sich schichtenweise. Kultur-historisches und philosophisches Wissen erleichtert das Lesen seiner Arbeiten, lässt hinter dem Dargestellten eine Welt der Gedanken, eine zutiefst menschliche Geisteshaltung und mehrere Ebenen von Deutungsmöglichkeiten erkennen. Rosenthal agiert «zwischen den Welten» ${ }^{34}$, schafft gewissermaßen eine «Malerei zwischen den Zeilen».

Der Argumentation des Kulturhistorikers Jost Hermand folgend, der die erklärt apolitischen Werke von Neo Rauch ${ }^{35}$ als «Vorboten einer möglichen Renaissance politischer Denkbilder ${ }^{36}$ bezeichnete, sind die Gemälde von Simon Rosenthal nicht zuletzt durch die zahlreichen darin enthaltenen Bezüge, durch ihren Subtext, politische Denkbilder.

Mehr als das - sie beziehen sich auf Politik und wirken aus sich heraus selbst politisch. Sie fordern zum Dialog, zum Meinungsstreit. Mit diesen sehr individuellen Kommentaren zum Zeitgeschehen, die der Künstler mit seinen Arbeiten in der Öffentlichkeit zur Diskussion stellt, mischt er sich bewusst in aktuelle gesellschaftliche Debatten ein. Während er die Gegenwart analysiert, blickt er in die Vergangenheit und entwirft zugleich eine Vision der Zukunft. Vielmehr als um Bilder der Macht geht es in seinen Werken, um die Macht der Bilder. Rosenthal erweist sich als Querdenker, betätigt sich im besten aristotelischen Sinne als «homo politicus», leistet seinen Beitrag zum gesellschaftlichen Diskurs.

Die Frage danach, wer wir sind, lässt der Künstler bewusst unbeantwortet - Rosenthal zeigt mögliche Wege und verweigert eindeutige Antworten. Seine Gemälde wirken auf einer Meta-Ebene. Sie motivieren, über unser Verhalten, über unser Verhältnis zur Welt nachzudenken, Nichts mehr und Nichts

33. Siehe Konrad Paul Liessmann: Rede zur Eröffnung der Salzburger Festspiele, Salzburg, 27.07.2016, veröffentlicht unter: http://www.salzburg.com/nachrichten/spezial/festspiele/salzburger-festspiele/sn/ artikel/rede-zur-eroeffnung-der-salzburger-festspiele-von-konrad-paul-liessmann-im-wortlaut-206882/ (04.08.2017).

34. So wählt er als Überschrift für einen seiner Texte bewusst «In between realities» - siehe RosENTHAL, In between realities.

35. Siehe auch Interview mit Neo Rauch, anlässlich der Filmpremiere im März 2017: Hans JoACHIM MÜLLER: «Schlaflose Nächte gehören bei mir ins Programm», in welt.de, 04.03.2017 - https://www.welt. $\mathrm{de} /$ kultur/kunst-und-architektur/article162581599/Schlaflose-Naechte-gehoeren-bei-mir-ins-Programm. html (05.08.2017).

36. Jost Hermand: Politische Denkbilder. Von Caspar David Friedrich bis Neo Rauch, böhlau, Wien, Köln, Weimar, 2011, S. 269. 
weniger. Der Künstler will mit seinen Werken weder die Welt erklären, noch den Betrachter belehren, sondern einen Impuls weitergeben: aufmerksam und vorurteilsfrei durch das Leben zu gehen, menschlich zu sein und zu bleiben. So gesehen schlummert vor allem in den narrativen Gemälden von Simon Rosenthal ein lebendiger Impetus.

\section{LITERATURVERZEICHNIS}

Salman Ansari (Hg.): Wolfgang Hildesheimer - Die Schule des Sehens, Insel, Frankfurt/Main, 1997, o. S.

Jörg Bernig: Habe Mut ... Eine Einmischung, 3. Kamenzer Rede in St. Annen, im Rahmen der Lessing-Rezeption, Kamenz, 07.09.2016, http://www.nachdenkseiten.de/upload/ pdf/161219-04-Endfassung-J-Bernig-3-Kamenzer-Rede.pdf (06.08.2017).

Sebastian Brant: Das Narrenschiff, Basel, 1494, Sächsische Landesbibliothek - Staats- und Universitätsbibliothek Dresden, Ink.394.4., http://digital.slub-dresden.de/werkansicht/ cache.off?tx_dlf\%5Bid\%5D $=11823 \&$ tx_dlf $\% 5$ Bpage $\% 5 \mathrm{D}=1 \& \mathrm{tx}$ _dlf $\% 5$ Bpointer $\% 5 \mathrm{D}=0$ (04.08.2017).

Paul Celan: «Niemandsrose», in Beda Allemann und Stefan Reichert (Hg.): Paul Celan - Gesammelte Werke in sieben Bänden, Suhrkamp, Frankfurt/Main, 1983, Bd. I, S. 211-291.

Joseph FreiherR von EICHENDORFF: "Wünschelrute», in AdAlbert von Chamisso und Gustav Schwab (Hg.): Deutscher Musenalmanach für das Jahr 1838, Weidmannsche Buchhandlung, Leipzig, 1838, S. 287, https://www.uni-due.de/lyriktheorie/scans2/1838_ eichendorff2.pdf (11.08.2017)

Michel FoucAult: Archäologie des Wissens, Suhrkamp, Frankfurt am Main, 1973 (L'archéologie du savoir, 1969).

Michel Foucault: Die Ordnung der Dinge: Eine Archäologie der Humanwissenschaften, Suhrkamp, Frankfurt am Main, 1971 (Les mots et les choses: Une archéologie des sciences humaines, 1966).

Marlies Giebe und Maria Körber: «... weil Dix hier wie ein Alter Meister und dabei doch ganz er selbst geblieben ist. Maltechnische Studien zum Triptychon „Der Krieg“ von Otto Dix», in Staatliche Kunstsammlungen Dresden (Hg.): Otto Dix. Der Krieg - Das Dresdner Triptychon, Katalog der Ausstellung, Staatliche Kunstsammlungen Dresden, Galerie Neue Meister Albertinum, Sandstein Verlag, Dresden, 2014, S. 219-251.

Jost Hermand: Politische Denkbilder. Von Caspar David Friedrich bis Neo Rauch, böhlau, Wien, Köln, Weimar, 2011, S. 269.

Hochschule Für Bildende Künste (Hg.): Diplom 2016, Katalog zur Ausstellung, 15.07.-04.09.2016, Dresden, 2016

IMMANUEL KANT: «Beantwortung der Frage: Was ist Aufklärung?», in Berlinische Monatsschrift, 1784, H. 12, S. 481- 494, http://www.deutschestextarchiv.de/book/view/ kant_aufklaerung_1784?p=17 (06.08.2017).

Konrad Paul Liessmann: Rede zur Eröffnung der Salzburger Festspiele, Salzburg, 27.07.2016, http://www.salzburg.com/nachrichten/spezial/festspiele/salzburger-festspiele/sn/ artikel/rede-zur-eroeffnung-der-salzburger-festspiele-von-konrad-paul-liessmann-imwortlaut-206882/ (04.08.2017).

Volker Maisel: «Vorwort», in Simon Rosenthal. Malerei und Grafik, Dresden, 2014, o. S.

Herman Melville: Moby-Dick or The Wale, Harper \& Brothers Publishers, New York, 1851 (Erstausgabe), http://www.gasl.org/refbib/Melville_Moby_Dick.pdf (05.08.2017).

Hans JoAChim Müller: «Schlaflose Nächte gehören bei mir ins Programm», in welt. de, 04.03.2017 - https://www.welt.de/kultur/kunst-und-architektur/article162581599/ Schlaflose-Naechte-gehoeren-bei-mir-ins-Programm.html (05.08.2017). 
KARIN MüLLER-Kelwing: «Ein neuer Ort für junge Kunst in Dresden. Zehn Künstler erfüllen sich ihren Traum», in Sachsenbummel. Magazin für KulturGeschichte und Tourismus, 23. Jg., Nr. 90, H. 1, 2016, S. 30-37.

Karin Müller-Kelwing: «Simon Rosenthal: Plateau Europa», in Hochschule für Bildende KüNSte (Hg.): Diplom 2016, Katalog zur Ausstellung, 15.07.-04.09.2016, Dresden, 2016, S. 145.

Gotthold Ephraim Lessing: Nathan der Weise. Ein dramatisches Gedicht, in fünf Aufzügen, Erstausgabe: Berlin 1779, http://gutenberg.spiegel.de/buch/nathan-der-weise-1179/3 (06.08.2017).

Nähe und Distanz. Internationale Ausstellung Skopje - Dresden, 11.09.-17.09.2016, Skopje, https://www.youtube.com/watch?v=8-YfGPSxM-w (04.08.2017).

Friedrich Nietzsche: Der Wille zur Macht. Versuch einer Umwertung aller Werte, Alfred Kröner Verlag, Leipzig, 1928.

GeORge Orwell: Nineteen Eighty-Four, Penguin, London 2008 (Erstausgabe 1948).

DoreEn ReInHARD: «Tumultartige Trauerarbeit», in Die ZEIT, 04.02.2017, http://www.zeit.de/ gesellschaft/zeitgeschehen/2017-02/dresden-gedenken-13-februar-tumulte-einweihungskulptur-monument-manaf-halbouni (04.08.2017).

Rainer Maria Rilke: «Archaïscher Torso Apollos», in Rainer Maria Rilke: Der neuen Gedichte anderer Teil, Insel-Verlag, Leipzig, 1918, S. 1, http://www.literature.at/viewer.alo? objid $=12096 \&$ viewmode $=$ fullscreen \&scale $=3.33 \&$ rotate $=\&$ page $=3(01.08 .2017)$.

Simon RosentHAL: In between realities, http://artitious.com/artist/simon-rosenthal/ (06.08.2017).

SimON Rosenthal: Soldat, 2016, http://atelier-simon-rosenthal.de/de/texts/ (05.08.2017).

Uwe Salzbrenner: «Kunst kommt um den Spuk aus Europa nicht herum. Absolventen der Hochschule für Bildende Künste zeigen ihre Diplomarbeiten», in Sächsische Zeitung, 18.07.2016, S. 19.

Holger Peter Saupe: «Dix-Schüler. Nähe und Distanz», in Kunstsammlung Gera (Hg.): Dix-Schüler. Nähe und Distanz. Gemälde Druckgraphiken Zeichnungen, Katalog der Ausstellung, Kunstsammlung Gera, 01.07.-20.08.1995, Gera 1995, S. 7-13.

G. Söder: «Dix an der Dresdner Kunstakademie», in Kunstsammlung Gera (Hg.): DixSchüler. Nähe und Distanz. Gemälde Druckgraphiken Zeichnungen, Katalog der Ausstellung, Kunstsammlung Gera, 01.07.-20.08.1995, Gera 1995, S. 39-46.

«Tumult auf dem Neumarkt», in Sächsische Zeitung, online, 07.02.2017, http://www.sz-online. de/nachrichten/tumult-auf-dem-neumarkt-3607272.html_(04.08.2017).

HeinZ Weissflog: «Das Prinzip Hoffnung. Malerei und Zeichnung von Simon Rosenthal in der produzentengalerie», in Dresdner Neueste Nachrichten, Nr. 286, 08.12.2016, S. 10.

http://atelier-simon-rosenthal.de/ (05.08.2017).

http://www.produzenten.net/ (10.08.2017).

https://de.wikipedia.org/wiki/Manipulation (05.08.2017).

https://de.wikisource.org/wiki/Der_Fischer (05.08.2017).

https://www.manaf-halbouni.com/work/monument/ (04.08.2017). 